\title{
Ubiquitination and deubiquitination of MCL1 in cancer: deciphering chemoresistance mechanisms and providing potential therapeutic options
}

\author{
Xiaowei Wu (1), Qingyu Luo (1) and Zhihua Liu (1)
}

\begin{abstract}
MCL1 is an important antiapoptotic member of the BCL-2 family that is distinguishable from other family members based on its relatively short half-life. Emerging studies have revealed the crucial role of MCL1 in the chemoresistance of cancer cells. The antiapoptotic function of MCL1 makes it a popular therapeutic target, although specific inhibitors have begun to emerge only recently. Notably, emerging studies have reported that several E3 ligases and deubiquitinases modulate MCL1 stability, providing an alternate means of targeting MCL1 activity. In addition, the emergence and development of proteolysis-targeting chimeras, the function of which is based on ubiquitinationmediated degradation, has shown great potential. In this review, we provide an overview of the studies investigating the ubiquitination and deubiquitination of MCL1, summarize the latest evidence regarding the development of therapeutic strategies targeting MCL1 in cancer treatment, and discuss the promising future of targeting MCL1 via the ubiquitin-proteasome system in clinical practice.
\end{abstract}

\section{Facts}

1. MCL1 is an important antiapoptotic member of the BCL-2 family, and the elevation of MCL1 protein level leads to chemoresistance and correlates with poor prognosis of cancer patients.

2. MCL1 is an unstable protein, and its stability is regulated by the ubiquitin-proteasome system (UPS).

3. Targeting MCL1 appears to be a promising strategy in cancer therapy, but the development of an effective inhibitor targeting MCL1 has begun only recently.

4. Targeting deubiquitinases upstream of MCL1 provides an alternative strategy for inhibiting MCL1 activity.

\section{Open questions}

1. What are the distinctions and relationships of the various E3 ligases/deubiquitinases that modulate MCL1?

\footnotetext{
Correspondence: Zhihua Liu (liuzh@cicams.ac.cn)

${ }^{1}$ State Key Laboratory of Molecular Oncology, National Cancer Center/National Clinical Research Center for Cancer/Cancer Hospital, Chinese Academy of Medical Sciences and Peking Union Medical College, 100021 Beijing, China These authors contributed equally: Xiaowei Wu, Qingyu Luo

Edited by M. Agostini
}

2. Is there a need to identify other E3 ligases or deubiquitinases that modulate MCL1?

3. Can the emerging strategy of targeting deubiquitinases upstream of MCL1 soon be successfully applied in clinical practice?

4. Is the use of a proteolysis-targeting chimera a promising strategy to directly target MCL1 by utilizing the UPS?

\section{Introduction}

Programmed cell death (PCD), a cell suicide process, is essential for normal organ development and tissue homeostasis. However, overactivation or inactivation of the PCD cascade can lead to pathogeneses, such as Parkinson's disease and tumorigenesis. Apoptosis is the predominant type of $\mathrm{PCD}$, whereas other types have also been discovered in recent years, such as pyroptosis ${ }^{1}$, necroptosis $^{2}$, parthanatos ${ }^{3}$, and ferroptosis ${ }^{4}$. Two distinct pathways can lead to apoptosis, the extrinsic/cell death receptor pathway and the intrinsic/mitochondrial pathway ${ }^{5}$. In the intrinsic pathway, B cell lymphoma 2 (BCL-2)

\section{(c) The Author(s) 2020}

(c) (i) Open Access This article is licensed under a Creative Commons Attribution 4.0 International License, which permits use, sharing, adaptation, distribution and reproduction c. in any medium or format, as long as you give appropriate credit to the original author(s) and the source, provide a link to the Creative Commons license, and indicate if changes were made. The images or other third party material in this article are included in the article's Creative Commons license, unless indicated otherwise in a credit line to the material. If material is not included in the article's Creative Commons license and your intended use is not permitted by statutory regulation or exceeds the permitted use, you will need to obtain permission directly from the copyright holder. To view a copy of this license, visit http://creativecommons.org/licenses/by/4.0/. 


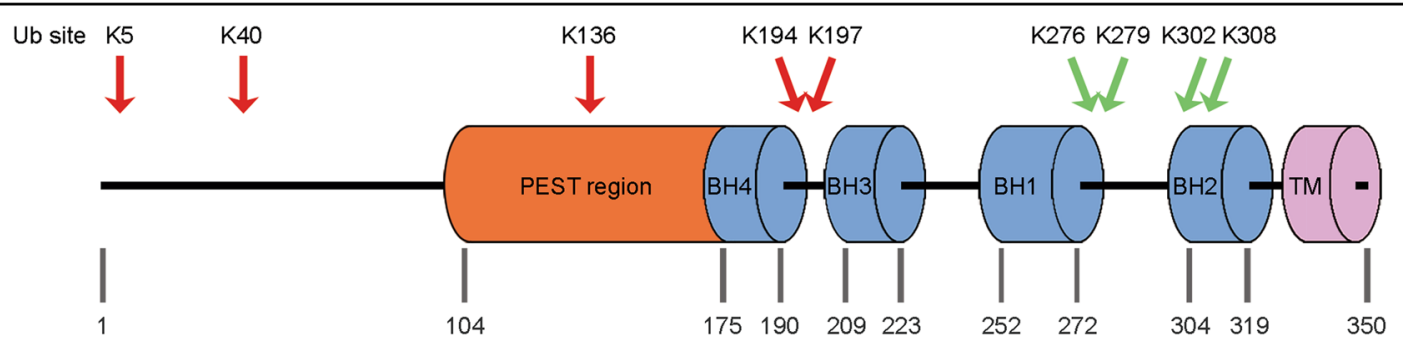

Fig. 1 Schematic model of MCL1 protein. MCL1 contains a long N-terminal regulatory domain harboring a PEST sequence, BH1-BH4 motifs and a C-terminal transmembrane (TM) domain. The arrows indicate the sites of ubiquitination (Ub): K48-linked polyubiquitin chains (red) and K63-linked polyubiquitin chains (green).

family members have been shown to have a critical role in regulating apoptosis by governing proapoptotic and antiapoptotic intracellular signals ${ }^{6}$. Numerous studies have revealed the subtle regulatory network between BCL-2 family members ${ }^{7,8}$, and their dysregulation can lead to various diseases ${ }^{9,10}$.

Chemotherapy is one of the most widely applied treatments for cancer in humans, and chemoresistance is one of the most severe obstacles that cancer patients face. Apoptosis escape is considered a hallmark of cancer ${ }^{11}$, contributing to the resistance of cancer cells to both chemotherapy $^{12,13}$ and radiotherapy ${ }^{14}$. Due to the regulatory role of the BCL-2 family in apoptotic signaling, studies of the BCL-2 family have elucidated the mechanisms underlying chemoresistance and provided several promising therapeutic strategies. Certainly, emerging attempts to target BCL-2 antiapoptotic members will benefit the treatment of cancer.

\section{Role of MCL1 in the mitochondrial apoptosis pathway}

The BCL2 family contains more than 25 proteins that regulate the intrinsic apoptosis pathway ${ }^{15}$. Based on their structure and function, BCL-2 family members are classified into three groups: antiapoptotic, proapoptotic, and proapoptotic BH3-only proteins. Antiapoptotic proteins that possess $\mathrm{BH} 1-4$ domains include MCL1, BCL-2, BCL-W, BCL-B, BCL-XL, and BFL-1/A1; proapoptotic proteins (apoptosis effectors) harboring BH1-4 domains include BAX and BAK; and proapoptotic BH3only proteins (apoptosis activators) include BIM, BID, BAD, NOXA, and PUMA ${ }^{16}$. Biochemical and structural studies have revealed that the $\mathrm{BH} 1, \mathrm{BH} 2$, and $\mathrm{BH} 3$ regions of antiapoptotic BCL-2 family proteins are proximal to one another and can form a surface-associated hydrophobic groove, a feature allowing interaction with the $\mathrm{BH} 3$ amphipathic helix of $\mathrm{BH} 3$-containing proapoptotic proteins and physiological antagonization ${ }^{15,17}$. MCL1 is a crucial antiapoptotic member of the BCL-2 family that was initially identified as an immediate-early gene expressed during the 12-O-tetra-decanoylphorbol-13- acetate (TPA)-mediated differentiation of the ML-1 human myeloid leukemia cell line ${ }^{18}$. MCL1 contains two PEST domains, four $\mathrm{BH}$ domains, and a C-terminal transmembrane (TM) domain as well as an unusually long $\mathrm{N}$ terminus that is not observed in other BCL-2 family members $^{19}$ (Fig. 1). The $\mathrm{N}$ terminus of MCL1 harbors numerous regulatory residues responsible for caspase cleavage, phosphorylation, and ubiquitination, and contains a PEST motif, a characteristic sequence of rapidly degraded proteins ${ }^{20}$. In addition, the BH1 domain and Cterminal region from aa 303 to 350 are also important for the rapid degradation of MCL1, contributing to its short half-life together with the PEST sequence ${ }^{21}$. The short MCL1 half-life distinguishes it from many other BCL-2 homologs ${ }^{22}$, and allows cells to rapidly switch between survival and apoptotic states in response to various stress signals. Another characteristic of MCL1 is its distinct tissue distribution and expression patterns compared with those of other BCL-2 family members ${ }^{23}$.

Several studies have provided a relatively detailed mechanism underlying the antiapoptotic function of MCL1. Classically, the MCL1 protein has been shown to anchor itself to the cytoplasmic face of the mitochondrial outer membrane via its C-terminal TM domain ${ }^{24}$, where it structurally interacts with and sequesters proapoptotic proteins to suppress mitochondrial outer membrane permeabilization (MOMP) and cytochrome $c$ release $\mathrm{e}^{25,26}$ (Fig. 2). Conversely, the above interactions can be antagonized by BH3-only proteins, which displace BAK and BAX from MCL1 to activate the mitochondrial apoptosis pathway ${ }^{6,27}$. In addition to the abovementioned classic antiapoptotic function of MCL1, maintenance of MCL1 levels has been shown to be necessary to preserve mitochondrial morphology and support normal mitochondrial bioenergetic activity in cardiomyocytes ${ }^{28,29}$. In addition, an amino-terminal truncated isoform of MCL1 has been reported to be anchored to the inner mitochondrial membrane (IMM) and exposed to the matrix where it retains the normal IMM structure, mitochondrial fusion, ATP production, membrane potential, and respiration ${ }^{30}$. This mitochondrial matrix form of MCL1 can also 


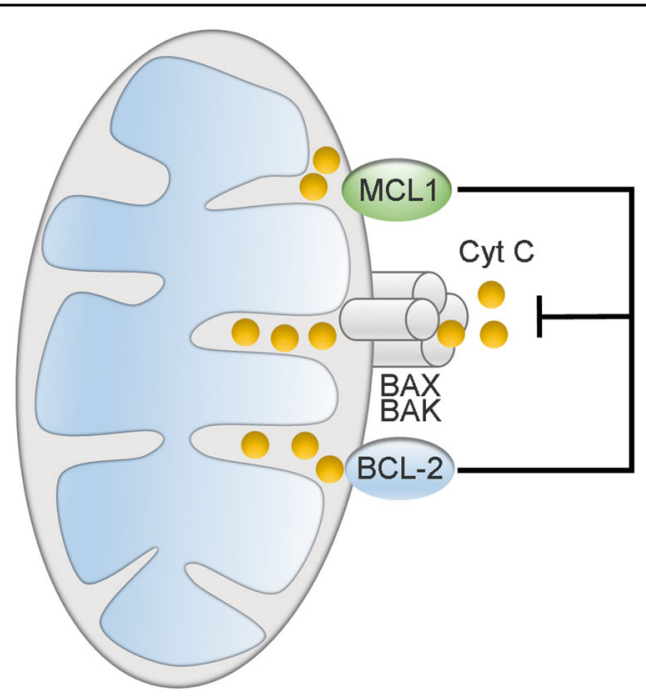

Fig. 2 The role of MCL1 in mitochondrial apoptotic signaling. MCL1 interacts with and sequesters proapoptotic proteins to suppress mitochondrial outer membrane permeabilization (MOMP) and cytochrome c (Cyt C) release to exert its pro-survival effects.

directly interact with and modulate very long-chain acylCoA dehydrogenase, a key enzyme in the mitochondrial fatty acid $\beta$-oxidation pathway, to engage in lipid metabolism $^{31}$. Excluding the functions within mitochondria, MCL1 can be translocated into the nucleus to activate Chk1 and maintain genome integrity in response to DNA damage $^{32,33}$. MCL1 also acts together with BCL-2 and $\mathrm{BCL}-\mathrm{XL}$ as a transcriptional regulator ${ }^{34}$ or as a stress sensor to participate in autophagy regulation ${ }^{35}$.

\section{Role of MCL1 in the context of cancer}

The amplification and elevated expression of MCL1 has been observed across cancer cell lines and human malignancies. A survey of the expression of antiapoptotic BCL-2 subfamily members in 68 human cancer cell lines revealed that MCL1 mRNA expression was higher than that of other BCL-2 members in lung, prostate, breast, ovarian, renal, and glioma cancer cell lines ${ }^{36}$. Increasing evidence has also shown that MCL1 is highly expressed in multiple cancer subtypes, including hematological malignancies $^{37}$, melanoma ${ }^{38}$, testicular germ cell tumor ${ }^{39}$, hepatocellular carcinoma ${ }^{40}$, breast cancer $^{22}$, urothelial carcinoma ${ }^{41}$ etc. Genetic studies have revealed that the MCL1 gene is located within 1q21.2, one of the most frequently amplified chromosome regions, and amplified in more than $10 \%$ of cancers ${ }^{42,43}$.

Because MCL1 functions as an efficient brake in the mitochondrial apoptosis pathway, it is understandable why MCL1 expression is preferentially increased in cancer cells to sustain their survival in response to various stresses, such as oncogenic stress, X-rays, chemotherapy, and small-molecule inhibitors ${ }^{44-46}$. Indeed, cancer patients with high levels of MCL1 expression have been shown to encounter drug resistance, relapse and poor prognosis outlook. For example, in diffuse large B cell leukemia, AKT-induced aerobic glycolysis promotes MCL1 protein synthesis, thereby maintaining cell survival and resistance to $\mathrm{BCL}-2$ inhibitors ${ }^{47}$. MCL1 is also frequently upregulated in breast cancer ${ }^{48}$, especially in drugresistant triple-negative breast cancer (TNBC) after neoadjuvant chemotherapy, with MCL1 (54\%) and MYC (35\%) gene coamplifications ${ }^{49}$. Elevated MCL1 expression has also been detected in chemoresistant cell lines and patients with ovarian cancer ${ }^{50,51}$.

\section{MCL1 modulation by UPS}

Ubiquitin, a 76-residue polypeptide, is a highly stable and conserved protein ${ }^{52}$, and ubiquitin conjugation is achieved through a cascade of multiple enzymatic reactions catalyzed by three classes of enzymes, including E1 ubiquitin-activating enzymes, E2 ubiquitin-conjugating enzymes, and E3 ubiquitin ligases $(\mathrm{E} 3 \mathrm{~s})^{53}$. Eight residues (M1, K6, K11, K27, K29, K33, K48, and K63) within ubiquitin serve as attachment sites for the formation of polyubiquitin chains ${ }^{54}$. Proteins modified with K48-linked chains, the most abundant linkage type in cells, are typically degraded by the $26 \mathrm{~S}$ proteasome ${ }^{53}$. Non-K48 linkages are primarily involved in nondegradative functions, including cellular signaling, intracellular trafficking, DNA damage response, and chromatin remodeling ${ }^{52,55}$, although their roles are not well defined. The ubiquitination process can be reversed and edited by deubiquitinases (DUBs), which cleave ubiquitin linkages from the substrates to alter their stability or activity ${ }^{56}$. All cellular proteins can be ubiquitinated at least once during their lifetime ${ }^{57}$; therefore, the ubiquitin code permeates every space in the cell and is involved in regulating almost every biological process.

Considering the short half-life and unstable nature of MCL1, posttranslational regulation, especially by the ubiquitin-proteasome system (UPS), is an important mechanism by which high MCL1 expression is maintained in cancer. To date, at least six E3s (Mule, $\mathrm{SCF}^{\beta-\mathrm{TrCP}}, \mathrm{SCF}^{\mathrm{FBW}}$, TRIM17, $\mathrm{APC} / \mathrm{C}^{\mathrm{Cdc} 20}$, and $\left.\mathrm{FBXO} 4\right)$ have been shown to have a role in the ubiquitination of MCL1 ${ }^{58,59}$. The first study suggesting that MCL1 could be regulated by the UPS was published in 2002 but lacked detailed experimental insight ${ }^{60}$. In 2003, two research groups successively reported that the rapid turnover of MCL1 is mediated by the proteasome ${ }^{61,62}$. In 2005, the first molecule shown to directly regulate the ubiquitination of MCL1 was identified through biochemical fractionation of cell extracts ${ }^{63}$. MCL1 ubiquitin ligase E3 (Mule) is a $482-\mathrm{kDa}$ HECT domain-containing ubiquitin ligase that was named for its ability to ubiquitinate MCL1 and induce its degradation in vitro ${ }^{63,64}$. Mule specifically 
acts on MCL1, as no interactions occur between the BH3 domain of Mule and BCL-XL, BCL-2, or BAX ${ }^{63}$.

The ubiquitination-mediated degradation of MCL1 has been shown to be dependent on its phosphorylation ${ }^{58}$. $\mathrm{SCF}^{\beta-T r C P}$ ubiquitinates and destabilizes MCL1 in a GSK3-dependent manner ${ }^{65}$, whereas the phosphorylation of MCL1 in $\mathrm{SCF}^{\mathrm{FBW} 7}$-induced MCL1 degradation can be mediated by the phosphokinases GSK3, JNK, p38, CKII, or CDK1 depending on the cellular context ${ }^{66,67}$. TRIM17 is the fourth E3 ligase demonstrated to target MCL1 ${ }^{68}$. Interestingly, both the ubiquitination of MCL1 by TRIM17 and the association between MCL1 and TRIM17 have also been shown to be associated with the phosphorylation of MCL1 by GSK $3^{68}$. APC/C $\mathrm{C}^{\mathrm{Cdc} 20}$ has been shown to engage in the ubiquitination of MCL1 and to control MCL1 stability during mitosis ${ }^{69,70}$; however, existing evidence appears to be insufficient to support the hypothesis that $\mathrm{APC} / \mathrm{C}^{\mathrm{Cdc} 20}$ is a bona fide MCL1 E3 ligase. FBXO4 is the last MCL1 E3 ligase identified and is a specific F-box protein that promotes MCL1 degradation in lung cancer ${ }^{59}$. Intriguingly, Choi et al. ${ }^{71}$ showed that an E3 ligase, TRAF6, promoted the K63-linked polyubiquitination of MCL1 on the C terminus and prevented its degradation; these results differ from those of previous studies showing that E3 ligases conjugate MCL1 with K48-linked polyubiquitin chains to promote its degradation.

\section{Deubiquitinases of MCL1}

The discovery of ubiquitination events and E3s related to the critical antiapoptotic protein MCL1 revealed one aspect of its posttranslational modulation. Deubiquitination is the reverse process of ubiquitination, and the ubiquitination of MCL1 can be counteracted by specific DUBs. Therefore, the identification of DUBs regulating

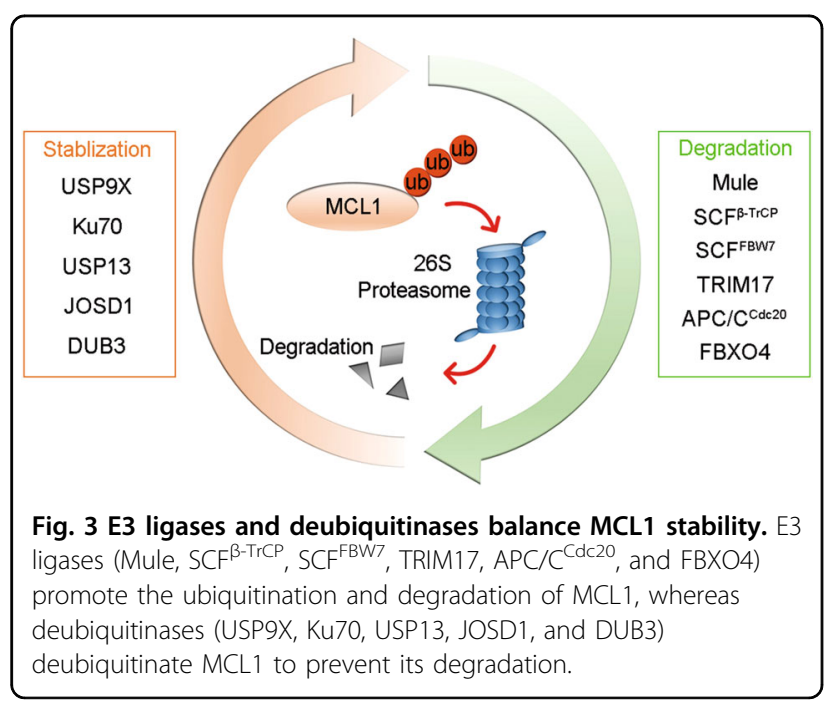

MCL1 revealed another side of this modulation process (Fig. 3).

\section{USP9X}

The first DUB targeting MCL1, USP9X, was identified among the immunoprecipitants interacting with MCL1 $1^{72}$. USP9X prolongs the half-life of MCL1 in an enzymatic activity-dependent manner by specifically cleaving the degradative K48-linked chains on MCL1 to prevent its proteasomal degradation ${ }^{72}$. Structurally, USP9X interacts with the $\mathrm{N}$ terminus of MCL1 but not with the BH domain, and the USP9X/MCL1 interaction is relatively weak compared with that between MCL1 and other BCL2 members, such as NOXA $^{72}$, indicating that this interaction may be easily disrupted. Indeed, subsequent studies showed that NOXA overexpression triggers a decrease in the USP9X/MCL1 interaction, leading to increased ubiquitination and degradation of MCL1 and the induction of apoptosis ${ }^{73,74}$. Schwickart et al. $^{72}$ also showed that maintaining the MCL1 residues Ser155, Ser159, and Thr163 in an unphosphorylated state is crucial for the interaction of USP9X and MCL1. Conversely, MCL1 phosphorylation at these sites by GSK3 $\beta$ disrupts the USP9X/MCL1 interaction, triggers its recognition by E3 ligases and promotes rapid degradation. It appears that phosphokinases regulate the state of MCL1 to determine whether it is degraded by its E3 ligases or stabilized by USP9X. Interestingly, USP9X exhibits biased expression in the brain and immune system ${ }^{75}$, and its function is dependent on the cancer subtype context. USP9X acts as an oncogene in multiple myeloma, lymphoma and nonsmall cell lung cancer ${ }^{72,74}$ but acts as a tumor suppressor in pancreatic ductal adenocarcinoma and colorectal cancer $^{76,77}$. Moreover, USP9X has also been reported to suppress colorectal cancer progression by stabilizing $\mathrm{SCF}^{\mathrm{FBW}}$, an MCL1 E3 ligase ${ }^{77}$.

\section{Ku70}

Lupus $\mathrm{Ku}$ autoantigen p70 (Ku70), a DNA doublestrand break (DSB) repair protein, has been shown to have an important role in the nonhomologous end-joining (NHEJ) pathway ${ }^{78}$. Intriguingly, in addition to its familiar function, Ku70 has been reported to possess intrinsic deubiquitination activity, cleaving degradative K48-linked polyubiquitin chains from MCL1 and promoting its stabilization $^{79}$. The role of Ku70 as a DUB has been further confirmed by evidence showing that Ku70 directly deubiquitinates MCL1 in a dose-dependent manner and hydrolyzes polyubiquitin chains into monoubiquitin units in vitro ${ }^{79}$. Ku70 specifically stabilizes MCL1 but none of the other antiapoptotic BCL-2 family members ${ }^{79}$. Interestingly, the $\mathrm{N}$ - and $\mathrm{C}$ termini of Ku70 have distinct functions as the $\mathrm{N}$ terminus (aa 1-535) exhibits DSB repair activity, and the $C$ terminus (aa 536-609) is 
required for the deubiquitination of MCL1 and the antiapoptotic activity of $\mathrm{Ku} 70^{79}$. In addition, IR enhances MCL1 nuclear translocation, increases the Ku70/MCL1 interaction and reduces the USP9X/MCL1 interaction in a dose-dependent manner ${ }^{79}$. This compensation strategy may be part of the mechanism underlying radioresistance in lung cancer.

\section{USP13}

USP13 was identified as a bone fide MCL1 DUB based on its ability to directly remove the ubiquitin chains modified on MCL1 in vitro ${ }^{80}$. Zhang et al. ${ }^{80}$ showed that both USP13 and MCL1 are genomically amplified in numerous cancer types and reported a positive correlation between USP13 and MCL1 in ovarian cancer patient tissues. However, a significant positive correlation between USP13 and MCL1 is not observable in lung cancer patient tissues $^{80}$, and our data even showed a negative correlation between USP13 and MCL1 in nine ovarian cancer cell lines $^{51}$. Previous studies have also shown that USP13 can deubiquitinate essential tumor suppressors (Vps34 complexes, p53, and PTEN) to function as a tumor suppressor in ovarian, breast and bladder cancers ${ }^{81-83}$. Thus, the significance of USP13-MCL1 regulation in the detailed context of cancer must be validated, and the possibility of targeting USP13 as a cancer treatment option must be explored.

\section{JOSD1}

JOSD1 was initially observed to exhibit membranerelated functions ${ }^{84}$, and a subsequent study revealed its antiviral effects via modulation of $\mathrm{SOCS}^{85}$. We performed an in vivo screen to identify essential DUBs that may contribute to the acquired chemoresistance of gynecological cancer, and the results revealed JOSD1 to be a key antiapoptotic DUB whose accumulation leads to acquired chemoresistance in both cervical and ovarian cancer. Our following studies confirmed that JOSD1 acts as a bona fide DUB that cleaves K48-linked polyubiquitin chains on MCL1 and protects it from UPS-mediated degradation. Interestingly, previous studies primarily revealed the role of JOSD1 on the cell membrane and proposed that the deubiquitination activity of JOSD1 is dependent on its monoubiquitination status ${ }^{84}$. In our in vitro deubiquitination assay, we observed that JOSD1 functioned as an MCL1 DUB, and that its deubiquitination activity was independent of its monoubiquitination ${ }^{86}$. Moreover, the colocalization of JOSD1 and MCL1 in the cytoplasm indicates that the DUB activity of JOSD1 may be more important in the cytoplasm than on the membrane, although relatively high JOSD1 expression has been observed on the membrane. In future studies, the JOSD1-MCL1 regulatory axis should be validated in other cancer types.

\section{DUB3}

As mentioned above, each previously reported MCL1 DUB has a largely context-specific effect on MCL1 stability. Furthermore, Zhang et al. ${ }^{80}$ also indicated that several DUBs may affect the stability of MCL1, although they selected USP13 for further studies due to its high amplification rates. Therefore, we performed an unbiased DUB screening to identify the strongest regulator of MCL1. DUB3 was confirmed to be the predominant factor that regulates the stability of $M C L 11^{51}$. By analyzing the expression levels of MCL1 and the other DUBs in nine ovarian cancer cell lines, we observed that only DUB3, and not USP9X or USP13, showed a significant positive correlation with MCL1, further supporting our hypothesis that DUB3 is the crucial DUB that modulates MCL1 stability ${ }^{51}$. Moreover, since DUB3 is a veritable oncogene due to its ability to stabilize Snail, Geminin, Cdc25A, NRF2, BRD4, and Cyclin $\mathrm{A}^{87-93}$, targeting DUB3 may be a promising strategy in cancer treatment with potentially fewer side effects.

\section{Targeting MCL1 in cancer treatment}

The specific structure of MCL1 had rendered the design of specific MCL1 inhibitors difficult ${ }^{94}$. Fortunately, the emergence of some specific MCL1 inhibitors holds promise for interfering with MCL1, and some BH3 mimetics targeting MCL1 are already being investigated in clinical practice $^{95-98}$. Targeting upstream transcriptional regulators and DUBs of MCL1 has provided another approach to target MCL1. Proteolysis-targeting chimeras (PROTACs) also show a promising future for targeting MCL1 (Fig. 4).

\section{Development of direct MCL1 inhibitors}

As we introduced above, BH3-only proteins displace BAK and BAX from MCL1 to induce apoptosis; thus, drugs mimicking BH3-only proteins might overcome chemoresistance. Conformational analyses showed that the hydrophobic groove of MCL1 is more rigid than that of other BCL-2 family members, and that MCL1 requires a high affinity for its endogenous ligands, which renders the design of MCL1 inhibitors challenging and explains why less progress has been achieved in their development compared with inhibitors targeting other BCL-2 family members ${ }^{6}$. A-1210477 is the first bona fide BH3 mimetic that has been demonstrated to target MCL1, as it selectively binds to MCL1 and disrupts the MCL1-BIM interaction ${ }^{99}$. In 2016, Kotschy et al. ${ }^{95}$ developed the first specific MCL1 inhibitor, S63845, which showed a 20-fold higher affinity than the previously reported A-1210477. The effect of S63845 was shown to be ideal for hematological tumors in subsequent studies ${ }^{100,101}$, although the effects on solid tumors were less pronounced ${ }^{51,95}$. Subsequently, AMG 176, a first-in-class orally bioavailable 


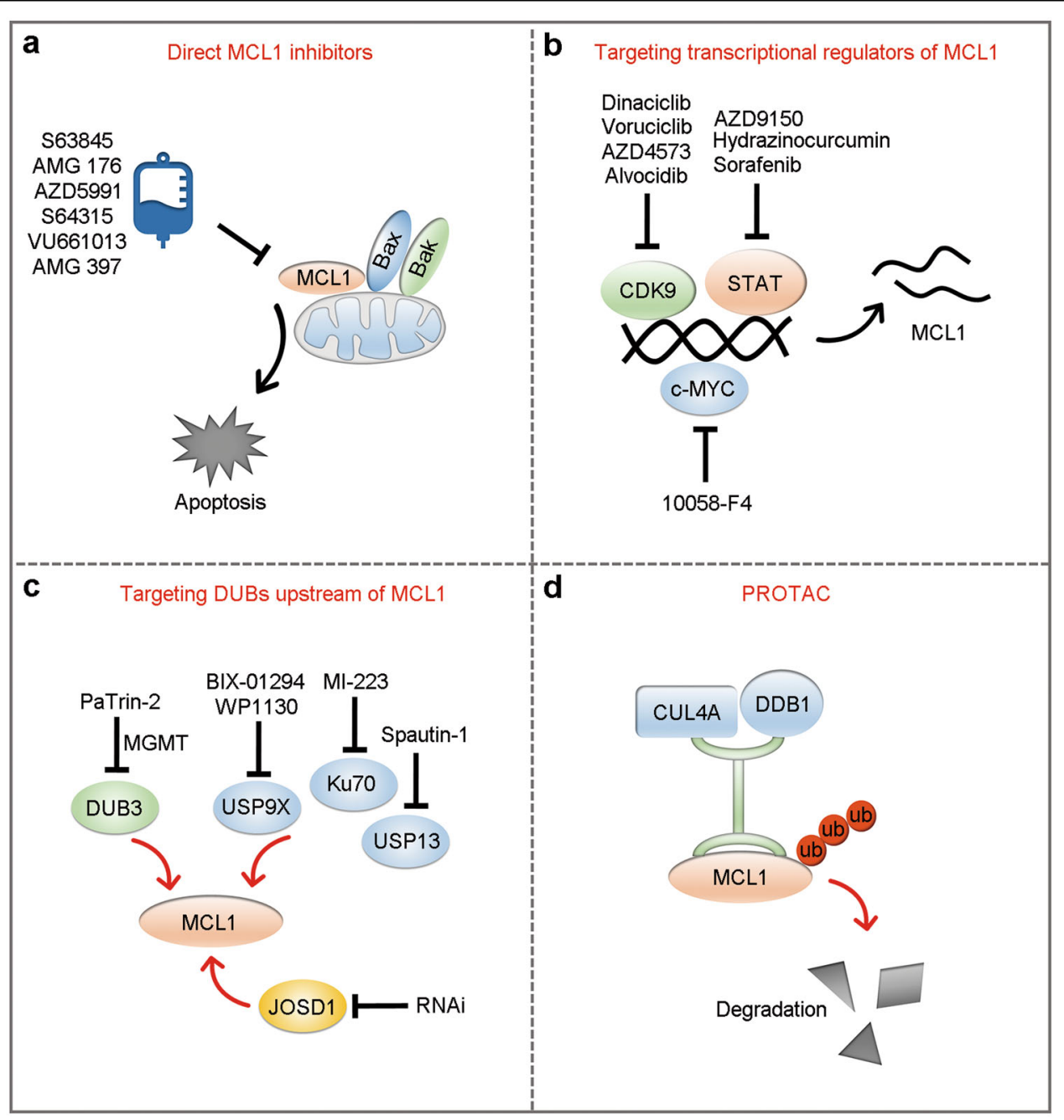

Fig. 4 Strategies for targeting MCL1. a Inhibitors specifically bind to MCL1, preventing its binding and inhibition of BAX and BAK, and leading to activation of mitochondrial apoptotic signaling. $\mathbf{b}$ Inhibitors indirectly suppress MCL1 by targeting transcriptional regulators of MCL1. $\mathbf{c}$ Inhibitors or RNAi indirectly suppress MCL1 by targeting MCL1 DUBs. $\mathbf{d}$ PROTAC strategy via recruitment of the CUL4A-DDB1 complex to facilitate ubiquitinationmediated degradation of MCL1 protein.

inhibitor of MCL1, was reported ${ }^{96}$. AMG 176 has two primary advantages over S63845: it can be administered orally and is effective against B cell, monocytic, and neutrophilic leukemia. Soon after the development of AMG 176, another MCL1 inhibitor named VU661013 was also reported ${ }^{98}$.

The sudden emergence of effective MCL1 inhibitors has broadened the horizon for strategies used to target MCL1, and studies have also confirmed the efficacy of using MCL1 inhibitors in combination with other BCL-2 family inhibitors ${ }^{98,102-104}$. Several MCL1 inhibitors, including $\mathrm{S}_{63845^{95}}$, AMG $176^{96}$, AZD5991 ${ }^{97}, \mathrm{~S} 64315^{105}$, and AMG 397 (https://clinicaltrials.gov), are already being investigated in the clinical stage, and the future of their application in the treatment of chemoresistance is encouraging. Notably, current MCL1 inhibitors show a higher affinity for human MCL1 than mouse MCL1, leading to complications for testing MCL1 inhibitors in pre-clinical mouse models. Fortunately, the recent generation of a humanized MCL1 mouse model provides a solution to more accurately evaluate the tolerability and efficacy of new MCL1 inhibitors in pre-clinical animal experiments $^{106}$.

\section{Targeting transcriptional regulators of MCL1}

As discussed above, the rigid structure and rapid turnover of MCL1 make the design of specific inhibitors against this protein difficult. Therefore, before the recent success of the development of specific MCL1 inhibitors, targeting the regulation of MCL1 was considered an easier strategy than developing an effective $\mathrm{BH} 3$ mimetic $^{107}$. One alternative strategy to target MCL1 is by facilitating 
its transcriptional repression. CDK9 has an important role in regulating gene transcription and has become a potential therapeutic target in cancer treatment ${ }^{108,109}$. Inhibition of CDK9 triggers the suppression of short-lived transcripts and proteins, such as MCL1, to induce tumor cell death $^{110,111}$, and is thus used in concert with BH3 mimetic drugs for cancer treatment ${ }^{112}$. Currently, the selective CDK9 inhibitors dinaciclib, voruciclib, AZD4573 and alvocidib are already under clinical development for the treatment of hematological malignancies ${ }^{110,111,113}$. Another category of MCL1 transcriptional regulator comprises the signal transducer and activator of transcription (STAT) transcription factors. Activation of either STAT1 ${ }^{114}$ or STAT3 ${ }^{115}$ can promote MCL1 transcription. FMS-like tyrosine kinase-3 signaling also activates MCL1 expression through its STAT5-docking domains ${ }^{116}$. Consistently, both suppression of STAT3 by an antisense oligonucleotide inhibitor (AZD9150) ${ }^{117}$ and inhibition of STAT3 phosphorylation by hydrazinocurcumin $^{118}$ lead to decreased MCL1 transcription. Combined usage of sorafenib (a multikinase inhibitor that suppresses STAT3 phosphorylation) and ABT-737 also synergize to induce glioma cell apoptosis by the inhibition of both MCL1 and BCL- ${ }^{119}$. c-MYC controls the transcription of MCL1 directly and also regulates BCL-XL by regulating the IF $4 E$ gene, in which context the inhibition of c-MYC sensitizes gastric cancer cells to the histone deacetylase inhibitor (HDACi) suberoylanilide hydroxamic $\operatorname{acid}^{120}$.

\section{Targeting DUBs upstream of MCL1}

The rapid discovery of several DUBs stabilizing MCL1 has provided another opportunity to indirectly target MCL1 by suppressing DUBs (Table 1). Indeed, USP9X knockdown decreases the level of MCL1 protein, sensitizes MCL1resistant colon carcinoma and leukemia cells to ABT- $737^{72}$, and sensitizes radioresistant cells to apoptosis induction ${ }^{121}$. The shRNA-mediated silencing of USP9X increases the sensitivity of chronic myelogenous leukemia cells to imatinib and other apoptotic stimuli ${ }^{122}$. BIX-01294, a euchromatic histone-lysine $N$-methyltransferase 2 inhibitor, has been shown to suppress the USP9X-MCL1 axis and induce apoptosis in bladder cancer cells ${ }^{123}$. WP1130, a secondgeneration tyrphostin derivative, was first identified in screens for AG490-like molecules ${ }^{124}$. Interestingly, WP1130 has also been confirmed to be a small-molecule inhibitor of USP9X activity ${ }^{125}$, and has been shown to trigger apoptosis in myeloid leukemia cells by destabilizing MCL1 ${ }^{122,124,125}$.

The discovery of Ku70 stabilizing MCL1 in lung cancer is also promising for the development of corresponding inhibitors. Chen et al. ${ }^{126}$ identified a novel small molecule, MI-223, that strongly synergizes with DNA replication stress agents to combat lung cancer by disrupting the MCL1/Ku70 interaction and inhibiting homologous
Table 1 Strategies for targeting DUBs upstream of MCL1 in cancer treatment.

\begin{tabular}{lll}
\hline DUB & Strategy & Cancer type \\
\hline USP9X & BIX-01294; WP1130 & Hematological malignancies \\
Ku70 & MI-223 & Lung cancer \\
USP13 & Spautin-1 & Ovarian cancer; lung cancer \\
JOSD1 & AAV-shRNA & Gynecological cancer \\
DUB3 & PaTrin-2 & Ovarian cancer \\
\hline
\end{tabular}

recombination activity. In another comparative study, Wang et al. ${ }^{79}$ showed that compared with that in cells individually treated with Ku70 shRNA or USP9X shRNA, the level of MCL1 in Ku70/USP9X double-knockdown cells was almost undetectable, increasing the sensitivity of lung cells to treatment with staurosporine or the BCL-2 inhibitor ABT-737. Thus, the development of an inhibitor targeting both Ku70 and USP9X, or at least a combination of both Ku70 and USP9X inhibitors, is a feasible approach for further studies. Interestingly, Zhang et al. $^{80}$ showed that pharmacological inhibition of USP13 by a smallmolecule inhibitor, spautin-1, markedly downregulated MCL1 protein levels and sensitized ovarian and lung cancer cells to ABT-263, a selective antagonist of BCL-2 and BCL-XL.

We selected DUB3 as an ideal target in ovarian cancer due to its oncogenic functions in various cancer subtypes ${ }^{51,87-93}$. After screening several small-molecule inhibitors, we observed that PaTrin-2 showed the most effective suppression of DUB3 mRNA expression ${ }^{51}$. Mechanistically, PaTrin-2 acts as a pseudosubstrate of $\mathrm{O}^{6}$-methylguanine-DNA methyltransferase (MGMT) ${ }^{127}$, and the inhibition of DUB3 transcription may result from the inhibition of its transcription factors by MGMT interference ${ }^{128}$. Functional study results verified that PaTrin-2 effectively reduced the viability of ovarian cancer cells with high MGMT-DUB3-MCL1 expression levels ${ }^{51}$. Most surprisingly, we observed that HDACis could activate DUB3, although the underlying mechanisms remain poorly understood. This "side effect" results in the successful use of PaTrin-2 and HDACis in combination for the treatment of ovarian cancer ${ }^{51}$. Of course, further clinical studies are still necessary to validate this effect. The rapid development of gene therapy has provided flexible methods for modulating the DUBs that stabilize MCL1. However, DUB3 is excluded from this approach because of its high level of homology with several members of the ubiquitin-specific peptidase 17-like family (USP17L1, USP17L3, USP17L4, and USP17L8) since "offtarget" effects would be difficult to avoid. For this reason, JOSD1 appears to be the most ideal gene therapy target. Using siRNA-mediated JOSD1 knockdown, we confirmed 
that the loss of JOSD1 could markedly increase the apoptosis of gynecological cancer cells ${ }^{86}$. Based on this evidence, adeno-associated virus (AAV)-mediated depletion of JOSD1 by shRNA was applied to several ovarian cancer patient-derived xenograft models, and the results showed that AAV treatment targeting JOSD1 significantly suppressed the growth of the xenografts ${ }^{86}$. The results of our study thus provide a promising strategy for the use of therapeutic gene-targeting methods to target DUBs upstream of MCL1.

\section{Application of PROTACs}

The UPS is an evolutionarily conserved apparatus in eukaryotes that is responsible for more than $80 \%$ of cellular proteolysis ${ }^{129}$. However, $~ 80 \%$ of proteins in human cells cannot be targeted pharmacologically and are defined as "undruggable"130. PROTACs are heterobifunctional molecules capable of bringing a target protein into spatial proximity with an E3 ubiquitin ligase and forming a target-PROTAC-ligase ternary complex to mediate target protein degradation by the UPS ${ }^{131,132}$. The first oral PROTAC drug ARV-110, which targets the androgen receptor for degradation in metastatic castration-resistant prostate cancer patients, was approved by the FDA for phase I clinical trials in $2019^{133}$. As expected, a recent study developed novel MCL1targeting PROTACs that effectively bring MCL1 into proximity of the E3 ligase CUL4A-DDB1-CRBN, which labels MCL1 with ubiquitin chains for proteasomal degradation at nanomolar concentrations, resulting in activation of the mitochondrial apoptosis pathway ${ }^{134}$. Importantly, recent studies have shown that BCL-XL PROTAC degraders can direct BCL-XL to CRBN and VHL E3 ligase-mediated degradation while sparing platelets because of the weak expression of CRBN and VHL E3 ligases in human platelets ${ }^{135,136}$. PROTAC technology exhibits advantages of reducing on-target toxicity, which provides insight into minimization of undesirable cardiotoxicity, hepatotoxicity, and hematological toxicity caused by MCL1 depletion via exploration of specific E3 ligases that are rarely expressed in cardiomyocytes, hepatocytes, hematopoietic stem cells, lymphocytes, and neutrophils ${ }^{28,29,137,138}$. These studies have launched the era of the use of PROTACs to target MCL1 in MCL1dominant cancers, although further supporting studies are urgently needed.

\section{Limitations for targeting MCL1}

Targeting MCL1 has shown great potential in cancer treatment. However, it is noteworthy that MCL1 also has an important role in early embryonic development ${ }^{139}$ and in the survival of multiple normal cell lineages ${ }^{140}$. MCL1 is highly expressed in the myocardium and is crucial for mitochondrial homeostasis and the induction of autophagy in cardiomyocytes ${ }^{141}$. Deficiency of MCL1 in murine hearts leads to rapid and fatal cardiomyopathy ${ }^{28,29}$, and inhibition of MCL1 in human cardiomyocytes results in a severe contractile defect ${ }^{142}$. Furthermore, deletion of MCL1 triggers the loss of hematopoietic stem cells, lymphocytes, and neutrophils ${ }^{138,143-145}$. MCL1 also contributes to the maintenance of hepatic integrity in murine livers ${ }^{146}$, and its absence in murine hepatocytes causes chronic liver damage ${ }^{137}$ and hepatocarcinogenesis ${ }^{147}$. Based on these studies, MCL1 depletion may introduce potential unwanted cardiotoxicity, hepatotoxicity, and hematological toxicity, especially in combination with other cytotoxic drugs. Thus, there is a need to identify a therapeutic window in which cancer cells are more sensitive than normal cells to the loss of MCL1. Interestingly, several groups have found that the loss of a single allele of $M C L 1$ can kill c-MYC-driven lymphoma cells, an alteration that is well tolerated in normal cells ${ }^{45,148,149}$. This anti-tumor effect while sparing normal cells by partial MCL1 inhibition may allow the establishment of a therapeutic window for MCL1 inhibitors. In addition, improving therapeutic methods to specifically deliver the inhibitors to cancer tissues will be another option to reduce side effects on normal tissues.

\section{Concluding remarks}

Chemoresistance is a severe concern regarding the poor prognosis of cancer patients, and MCL1, an antiapoptotic BCL-2 family member, has become a popular target for cancer treatment due to its important effects. The stalled development of specific MCL1 inhibitors prompted studies of E3 ligases and DUBs modulating MCL1 and the development of their corresponding inhibitors. Considering the rapid turnover of MCL1 and its rigid structure, indirectly targeting MCL1 (e.g., via DUB inhibitors) may represent a promising alternative. Given the number of known MCL1 DUBs and those not yet identified, targeting only one MCL1 DUB seems unlikely to ensure a beneficial effect because the change in MCL1 protein levels may be compensated by other DUBs. Moreover, since DUBs are usually not monospecific for MCL1, offtarget toxicity and loss of therapeutic effects are possible in applications targeting DUBs. However, if the dominant DUB that controls MCL1 stability in a specific context can be identified, it would be a promising target. The additional tumor-suppressing substrates of DUB should also be considered. Thus, the reliability of targeting the USP9X-MCL1 and USP13-MCL1 axes should be further explored in specific cancer subtypes. Another potentially large liability of DUB inhibitors is that none have entered the clinical stage except for VLX1570, which targets UCHL5 and USP14, but this clinical trial has been 
suspended ${ }^{150}$. However, the development of DUB inhibitors is still very young, and numerous DUB inhibitors are in the pre-clinical stage ${ }^{150}$. Therefore, targeting DUBs upstream of MCL1 shows great potential in future research.

\section{Acknowledgements}

We apologize to the authors working on topics related to this review who were not included due to space constraints. This work was supported by the National Key R\&D Program of China (2016YFC1302100) and the CAMS Innovation Fund for Medical Sciences (2016-I2M-1-001, 2019-12M-1-003).

\section{Conflict of interest}

The authors declare that they have no conflict of interest.

\section{Publisher's note}

Springer Nature remains neutral with regard to jurisdictional claims in published maps and institutional affiliations.

Received: 16 March 2020 Revised: 3 July 2020 Accepted: 6 July 2020 Published online: 22 July 2020

\section{References}

1. Cookson, B. T. \& Brennan, M. A. Pro-inflammatory programmed cell death Trends Microbiol. 9, 113-114 (2001).

2. Degterev, A. et al. Chemical inhibitor of nonapoptotic cell death with therapeutic potential for ischemic brain injury. Nat. Chem. Biol. 1, 112-119 (2005)

3. Andrabi, S. A., Dawson, T. M. \& Dawson, V. L. Mitochondrial and nuclear cross talk in cell death: parthanatos. Ann. N. Y Acad. Sci. 1147, 233-241 (2008).

4. Dixon, S. J. et al. Ferroptosis: an iron-dependent form of nonapoptotic cell death. Cell 149, 1060-1072 (2012).

5. Elmore, S. Apoptosis: a review of programmed cell death. Toxicol. Pathol. $\mathbf{3 5}$ 495-516 (2007).

6. Ashkenazi, A., Fairbrother, W. J., Leverson, J. D. \& Souers, A. J. From basic apoptosis discoveries to advanced selective BCL-2 family inhibitors. Nat. Rev. Drug Discov. 16, 273-284 (2017).

7. Kale, J., Osterlund, E. J. \& Andrews, D. W. BCL-2 family proteins: changing partners in the dance towards death. Cell Death Differ. 25, 65-80 (2018).

8. Opferman, J. T. \& Kothari, A. Anti-apoptotic BCL-2 family members in development. Cell Death Differ. 25, 37-45 (2018).

9. Singh, R., Letai, A. \& Sarosiek, K. Regulation of apoptosis in health and disease: the balancing act of BCL-2 family proteins. Nat. Rev. Mol. Cell Biol. 20, 175-193 (2019).

10. Delbridge, A. R., Grabow, S., Strasser, A. \& Vaux, D. L. Thirty years of BCL-2: translating cell death discoveries into novel cancer therapies. Nat. Rev. Cancer 16, 99-109 (2016).

11. Hanahan, D. \& Weinberg, R. A. Hallmarks of cancer: the next generation. Cell 144, 646-674 (2011)

12. Skarkova, V., Kralova, V., Vitovcova, B. \& Rudolf, E. Selected aspects of chemoresistance mechanisms in colorectal carcinoma-A focus on epithelial-tomesenchymal transition, autophagy, and apoptosis. Cells $\mathbf{8}$, https:/doi.org/ 10.3390/cells8030234 (2019).

13. Luo, Q. et al. ARID1A ablation leads to multiple drug resistance in ovarian cancer via transcriptional activation of MRP2. Cancer Lett. 427, 9-17 (2018).

14. Barker, H. E., Paget, J. T., Khan, A. A. \& Harrington, K. J. The tumour microenvironment after radiotherapy: mechanisms of resistance and recurrence. Nat. Rev. Cancer 15, 409-425 (2015).

15. Petros, A. M., Olejniczak, E. T. \& Fesik, S. W. Structural biology of the BCl-2 family of proteins. Biochim. Biophys. Acta 1644, 83-94 (2004).

16. Happo, L., Strasser, A. \& Cory, S. BH3-only proteins in apoptosis at a glance. J. Cell Sci. 125, 1081-1087 (2012).

17. Kong, W. et al. Experimental characterization of the binding affinities between proapoptotic $\mathrm{BH} 3$ peptides and antiapoptotic $\mathrm{BCl}-2$ proteins. ChemMedChem 13, 1763-1770 (2018).
18. Kozopas, K. M., Yang, T., Buchan, H. L., Zhou, P. \& Craig, R. W. MCL1, a gene expressed in programmed myeloid cell differentiation, has sequence similarity to BCL2. Proc. Natl Acad. Sci. USA 90, 3516-3520 (1993).

19. Germain, M. \& Duronio, V. The $\mathrm{N}$ terminus of the anti-apoptotic BCL-2 homologue MCL-1 regulates its localization and function. J. Biol. Chem. 282, 32233-32242 (2007)

20. Rechsteiner, M. \& Rogers, S. W. PEST sequences and regulation by proteolysis. Trends Biochem Sci. 21, 267-271 (1996).

21. Mei, Y., Du, W., Yang, Y. \& Wu, M. Puma(*)Mcl-1 interaction is not sufficient to prevent rapid degradation of Mcl-1. Oncogene 24, 7224-7237 (2005).

22. Campbell, K. J. et al. MCL-1 is a prognostic indicator and drug target in breast cancer. Cell Death Dis. 9, 19 (2018).

23. Yang, T., Kozopas, K. M. \& Craig, R. W. The intracellular distribution and pattern of expression of $\mathrm{Mcl}-1$ overlap with, but are not identical to, those of $\mathrm{BCl}-2$. J. Cell Biol. 128, 1173-1184 (1995).

24. Akgul, C., Moulding, D. A., White, M. R. \& Edwards, S. W. In vivo localisation and stability of human Mcl-1 using green fluorescent protein (GFP) fusion proteins. FEBS Lett. 478, 72-76 (2000).

25. Chen, L. et al. Differential targeting of prosurvival $\mathrm{BCl}-2$ proteins by their $\mathrm{BH} 3-$ only ligands allows complementary apoptotic function. Mol. Cell 17, 393-403 (2005).

26. Green, D. R. \& Reed, J. C. Mitochondria and apoptosis. Science 281, 1309-1312 (1998).

27. Shamas-Din, A., Brahmbhatt, H., Leber, B. \& Andrews, D. W. BH3-only proteins: orchestrators of apoptosis. Biochim. Biophys. Acta 1813, 508-520 (2011).

28. Wang, $\mathrm{X}$. et al. Deletion of MCL-1 causes lethal cardiac failure and mitochondrial dysfunction. Genes Dev. 27, 1351-1364 (2013).

29. Thomas, R. L. et al. Loss of MCL-1 leads to impaired autophagy and rapid development of heart failure. Genes Dev. 27, 1365-1377 (2013).

30. Perciavalle, R. M. et al. Anti-apoptotic MCL-1 localizes to the mitochondrial matrix and couples mitochondrial fusion to respiration. Nat. Cell Biol. 14, 575-583 (2012).

31. Escudero, S. et al. Dynamic regulation of long-chain fatty acid oxidation by a noncanonical interaction between the MCL-1 BH3 helix and VLCAD. Mol. Cell 69. 729-743 e727 (2018)

32. Jamil, S., Mojtabavi, S., Hojabrpour, P., Cheah, S. \& Duronio, V. An essential role for MCL-1 in ATR-mediated CHK1 phosphorylation. Mol. Biol. Cell 19 3212-3220 (2008).

33. Pawlikowska, P. et al. ATM-dependent expression of IEX-1 controls nuclear accumulation of Mcl-1 and the DNA damage response. Cell Death Differ. 17, 1739-1750 (2010).

34. $\mathrm{Wu}, \mathrm{X}$. et al. Extra-mitochondrial prosurvival $\mathrm{BCL}-2$ proteins regulate gene transcription by inhibiting the SUFU tumour suppressor. Nat. Cell Biol. 19 1226-1236 (2017)

35. Germain, M. et al. MCL-1 is a stress sensor that regulates autophagy in a developmentally regulated manner. EMBO J. 30, 395-407 (2011).

36. Placzek, W. J. et al. A survey of the anti-apoptotic Bcl-2 subfamily expression in cancer types provides a platform to predict the efficacy of $\mathrm{BCl}-2$ antagonists in cancer therapy. Cell Death Dis. 1, e40 (2010).

37. Li, Q. F. et al. Sphingosine kinase-1 mediates BCR/ABL-induced upregulation of Mcl-1 in chronic myeloid leukemia cells. Oncogene 26, 7904-7908 (2007).

38. Lesinski, G. B. et al. IFN-alpha and bortezomib overcome $\mathrm{BCl}-2$ and $\mathrm{Mcl}-1$ overexpression in melanoma cells by stimulating the extrinsic pathway of apoptosis. Cancer Res. 68, 8351-8360 (2008).

39. Sano, M. et al. Overexpression of anti-apoptotic $\mathrm{Mcl}-1$ in testicular germ cell tumours. Histopathology 46, 532-539 (2005).

40. Sieghart, W. et al. Mcl-1 overexpression in hepatocellular carcinoma: a potential target for antisense therapy. J. Hepatol. 44, 151-157 (2006).

41. Hong, A. L. et al. MCL1 and DEDD promote urothelial carcinoma progression. Mol. Cancer Res 17, 1294-1304 (2019).

42. Beroukhim, R. et al. The landscape of somatic copy-number alteration across human cancers. Nature 463, 899-905 (2010).

43. Zack, T. I. et al. Pan-cancer patterns of somatic copy number alteration. Nat. Genet. 45, 1134-1140 (2013).

44. Evan, G. I. et al. Induction of apoptosis in fibroblasts by c-myc protein. Cell 69 119-128 (1992).

45. Grabow, S., Delbridge, A. R., Aubrey, B. J., Vandenberg, C. J. \& Strasser, A. Loss of a single Mcl-1 allele inhibits MYC-driven lymphomagenesis by sensitizing Pro-B cells to apoptosis. Cell Rep. 14, 2337-2347 (2016). 
46. van Delft, M. F. et al. The $\mathrm{BH} 3$ mimetic $\mathrm{ABT}-737$ targets selective $\mathrm{BCl}-2$ proteins and efficiently induces apoptosis via Bak/Bax if Mcl-1 is neutralized. Cancer Cell 10, 389-399 (2006).

47. Coloff, J. L. et al. Akt-dependent glucose metabolism promotes Mcl-1 synthesis to maintain cell survival and resistance to $\mathrm{BCl}-2$ inhibition. Cancer Res. 71, 5204-5213 (2011).

48. Glodzik, D. et al. Mutational mechanisms of amplifications revealed by analysis of clustered rearrangements in breast cancers. Ann. Oncol. 29, 2223-2231 (2018).

49. Balko, J. M. et al. Molecular profiling of the residual disease of triple-negative breast cancers after neoadjuvant chemotherapy identifies actionable therapeutic targets. Cancer Discov. 4, 232-245 (2014).

50. Wu, X. et al. JOSD1 inhibits mitochondrial apoptotic signalling to drive acquired chemoresistance in gynaecological cancer by stabilizing MCL1. Cell Death Differ. 27, 55-70 (2020).

51. Wu, X. et al. MGMT-activated DUB3 stabilizes MCL1 and drives chemoresistance in ovarian cancer. Proc. Natl Acad. Sci. USA 116, 2961-2966 (2019).

52. Komander, D. \& Rape, M. The ubiquitin code. Annu Rev. Biochem. 81, 203-229 (2012).

53. Hershko, A. \& Ciechanover, A. The ubiquitin system. Annu Rev. Biochem. 67, 425-479 (1998).

54. Swatek, K. N. \& Komander, D. Ubiquitin modifications. Cell Res. 26, 399-422 (2016).

55. Yau, R. \& Rape, M. The increasing complexity of the ubiquitin code. Nat. Cell Biol. 18, 579-586 (2016).

56. Komander, D., Clague, M. J. \& Urbe, S. Breaking the chains: structure and function of the deubiquitinases. Nat. Rev. Mol. Cell Biol. 10, 550-563 (2009).

57. Senft, D., Qi, J. \& Ronai, Z. A. Ubiquitin ligases in oncogenic transformation and cancer therapy. Nat. Rev. Cancer 18, 69-88 (2018).

58. Mojsa, B., Lassot, I. \& Desagher, S. Mcl-1 ubiquitination: unique regulation of an essential survival protein. Cells 3, 418-437 (2014).

59. Feng, C., Yang, F. \& Wang, J. FBXO4 inhibits lung cancer cell survival by targeting Mcl-1 for degradation. Cancer Gene Ther. 24, 342-347 (2017).

60. Gojo, I., Zhang, B. \& Fenton, R. G. The cyclin-dependent kinase inhibitor flavopiridol induces apoptosis in multiple myeloma cells through transcriptional repression and down-regulation of Mcl-1. Clin. Cancer Res. 8, 3527-3538 (2002).

61. Nijhawan, D. et al. Elimination of Mcl-1 is required for the initiation of apoptosis following ultraviolet irradiation. Genes Dev. 17, 1475-1486 (2003).

62. Cuconati, A., Mukherjee, C., Perez, D. \& White, E. DNA damage response and MCL-1 destruction initiate apoptosis in adenovirus-infected cells. Genes Dev. 17, 2922-2932 (2003)

63. Zhong, Q., Gao, W., Du, F. \& Wang, X. Mule/ARF-BP1, a BH3-only E3 ubiquitin ligase, catalyzes the polyubiquitination of $\mathrm{Mcl}-1$ and regulates apoptosis. Cell 121, 1085-1095 (2005).

64. Warr, M. R. et al. BH3-ligand regulates access of MCL-1 to its E3 ligase. FEBS Lett. 579, 5603-5608 (2005).

65. Ding, Q. et al. Degradation of $\mathrm{MCl}-1$ by beta-TrCP mediates glycogen synthase kinase 3-induced tumor suppression and chemosensitization. Mol. Cell Biol. 27, 4006-4017 (2007).

66. Millman, S. E. \& Pagano, M. MCL1 meets its end during mitotic arrest. EMBO Rep. 12, 384-385 (2011).

67. Wertz, I. E. et al. Sensitivity to antitubulin chemotherapeutics is regulated by MCL1 and FBW7. Nature 471, 110-114 (2011).

68. Magiera, M. M. et al. Trim17-mediated ubiquitination and degradation of Mcl-1 initiate apoptosis in neurons. Cell Death Differ. 20, 281-292 (2013).

69. Harley, M. E., Allan, L. A., Sanderson, H. S. \& Clarke, P. R. Phosphorylation of Mcl-1 by CDK1-cyclin B1 initiates its Cdc20-dependent destruction during mitotic arrest. EMBO J. 29, 2407-2420 (2010).

70. Allan, L. A., Skowyra, A., Rogers, K. I., Zeller, D. \& Clarke, P. R. Atypical APC/Cdependent degradation of $\mathrm{MCl}-1$ provides an apoptotic timer during mitotic arrest. EMBO J. 37, https://doi.org/10.15252/embj.201796831 (2018).

71. Choi, Y. B. \& Harhaj, E. W. HTLV-1 tax stabilizes MCL-1 via TRAF6-dependent K63-linked polyubiquitination to promote cell survival and transformation. PLoS Pathog. 10, e1004458 (2014).

72. Schwickart, M. et al. Deubiquitinase USP9X stabilizes MCL1 and promotes tumour cell survival. Nature 463, 103-107 (2010).

73. Gomez-Bougie, P. et al. Noxa controls Mule-dependent Mcl-1 ubiquitination through the regulation of the Mcl-1/USP9X interaction. Biochem. Biophys. Res. Commun. 413, 460-464 (2011).
74. Yan, J. et al. Usp9x- and Noxa-mediated Mcl-1 downregulation contributes to pemetrexed-induced apoptosis in human non-small-cell lung cancer cells. Cell Death Dis. 5, e1316 (2014).

75. Naik, E. et al. Regulation of proximal T cell receptor signaling and tolerance induction by deubiquitinase Usp9X. J. Exp. Med. 211, 1947-1955 (2014).

76. Perez-Mancera, P. A. et al. The deubiquitinase USP9X suppresses pancreatic ductal adenocarcinoma. Nature 486, 266-270 (2012).

77. Khan, O. M. et al. The deubiquitinase USP9X regulates FBW7 stability and suppresses colorectal cancer. J. Clin. Invest. 128, 1326-1337 (2018).

78. Downs, J. A. \& Jackson, S. P. A means to a DNA end: the many roles of Ku. Nat. Rev. Mol. Cell Biol. 5, 367-378 (2004).

79. Wang, B. et al. Role of Ku70 in deubiquitination of Mcl-1 and suppression of apoptosis. Cell Death Differ. 21, 1160-1169 (2014).

80. Zhang, S. et al. Deubiquitinase USP13 dictates MCL1 stability and sensitivity to BH3 mimetic inhibitors. Nat. Commun. 9, 215 (2018)

81. Zhang, J. et al. Deubiquitylation and stabilization of PTEN by USP13. Nat. Cell Biol. 15, 1486-1494 (2013).

82. Liu, J. et al. Beclin1 controls the levels of p53 by regulating the deubiquitination activity of USP10 and USP13. Cell 147, 223-234 (2011).

83. Man, X. et al. USP13 functions as a tumor suppressor by blocking the NF-kBmediated PTEN downregulation in human bladder cancer. J. Exp. Clin. Cancer Res. 38, 259 (2019).

84. Seki, T. et al. JosD1, a membrane-targeted deubiquitinating enzyme, is activated by ubiquitination and regulates membrane dynamics, cell motility, and endocytosis. J. Biol. Chem. 288, 17145-17155 (2013).

85. Wang, $X$. et al. JOSD1 negatively regulates type-l interferon antiviral activity by deubiquitinating and stabilizing SOCS1. Viral Immunol. 30, 342-349 (2017).

86. $\mathrm{Wu}, \mathrm{X}$. et al. JOSD1 inhibits mitochondrial apoptotic signalling to drive acquired chemoresistance in gynaecological cancer by stabilizing MCL1. Cell Death Differ. https://doi.org/10.1038/s41418-019-0339-0 (2019).

87. Pereg, Y. et al. Ubiquitin hydrolase Dub3 promotes oncogenic transformation by stabilizing Cdc25A. Nat. Cell Biol. 12, 400-406 (2010).

88. Liu, T. et al. CDK4/6-dependent activation of DUB3 regulates cancer metastasis through SNAIL1. Nat. Commun. 8, 13923 (2017).

89. Wu, Y. et al. Dub3 inhibition suppresses breast cancer invasion and metastasis by promoting Snail1 degradation. Nat. Commun. 8, 14228 (2017).

90. Jin, $X$. et al. DUB3 promotes BET inhibitor resistance and cancer progression by deubiquitinating BRD4. Mol. Cell 71, 592-605 e594 (2018).

91. Zhang, Q. et al. DUB3 deubiquitinates and stabilizes NRF2 in chemotherapy resistance of colorectal cancer. Cell Death Differ. 26, 2300-2313 (2019).

92. Hu, B. et al. Deubiquitinase DUB3 regulates cell cycle progression via stabilizing cyclin A for proliferation of non-small cell lung cancer cells. Cells $\mathbf{8}$, https://doi.org/10.3390/cells8040297 (2019).

93. Hernandez-Perez, S. et al. DUB3 and USP7 de-ubiquitinating enzymes control replication inhibitor Geminin: molecular characterization and associations with breast cancer. Oncogene 36, 4802-4809 (2017).

94. Arkin, M. R. \& Wells, J. A. Small-molecule inhibitors of protein-protein interactions: progressing towards the dream. Nat. Rev. Drug Discov. 3, 301-317 (2004).

95. Kotschy, A. et al. The MCL1 inhibitor $\mathbf{5 6 3 8 4 5}$ is tolerable and effective in diverse cancer models. Nature 538, 477-482 (2016)

96. Caenepeel, $\mathrm{S}$. et al. AMG 176, a selective MCL1 inhibitor, is effective in hematologic cancer models alone and in combination with established therapies. Cancer Discov. 8, 1582-1597 (2018).

97. Tron, A. E. et al. Discovery of Mcl-1-specific inhibitor AZD5991 and preclinical activity in multiple myeloma and acute myeloid leukemia. Nat. Commun. $\mathbf{9}$, 5341 (2018).

98. Ramsey, H. E. et al. A novel MCL1 inhibitor combined with venetoclax rescues venetoclax-resistant acute myelogenous leukemia. Cancer Discov. 8 1566-1581 (2018).

99. Leverson, J. D. et al. Potent and selective small-molecule MCL-1 inhibitors demonstrate on-target cancer cell killing activity as single agents and in combination with ABT-263 (navitoclax). Cell Death Dis. 6, e1590 (2015).

100. Moujalled, D. M. et al. Combining BH3-mimetics to target both BCL-2 and MCL1 has potent activity in pre-clinical models of acute myeloid leukemia. Leukemia 33, 905-917 (2019).

101. Li, Z., He, S. \& Look, A. T. The MCL1-specific inhibitor 563845 acts synergistically with venetoclax/ABT-199 to induce apoptosis in T-cell acute lymphoblastic leukemia cells. Leukemia 33, 262-266 (2019). 
102. Nangia, V. et al. Exploiting MCL1 dependency with combination MEK + MCL1 inhibitors leads to induction of apoptosis and tumor regression in KRAS-mutant non-small cell lung cancer. Cancer Discov. 8, 1598-1613 (2018).

103. Prukova, D. et al. Cotargeting of BCL2 with venetoclax and MCL1 with S63845 is synthetically lethal in vivo in relapsed mantle cell lymphoma. Clin. Cancer Res. 25, 4455-4465 (2019).

104. Anstee, N. S. et al. Impact of elevated anti-apoptotic MCL-1 and BCL-2 on the development and treatment of MLL-AF9 AML in mice. Cell Death Differ. 26, 1316-1331 (2019).

105. Szlavik, Z. et al. Structure-guided discovery of a selective Mcl-1 inhibitor with cellular activity. J. Med. Chem. 62, 6913-6924 (2019).

106. Brennan, M. S. et al. Humanized Mcl-1 mice enable accurate preclinical evaluation of MCL-1 inhibitors destined for clinical use. Blood $\mathbf{1 3 2}$ 1573-1583 (2018).

107. Czabotar, P. E., Lessene, G., Strasser, A. \& Adams, J. M. Control of apoptosis by the $\mathrm{BCL}-2$ protein family: implications for physiology and therapy. Nat. Rev. Mol. Cell Biol. 15, 49-63 (2014).

108. Lim, S. \& Kaldis, P. Cdks, cyclins and CKls: roles beyond cell cycle regulation. Development 140, 3079-3093 (2013).

109. Tibes, R. \& Bogenberger, J. M. Transcriptional silencing of MCL-1 through cyclin-dependent kinase inhibition in acute myeloid leukemia. Front. Oncol. $\mathbf{9}$ 1205 (2019).

110. Cidado, J. et al. AZD4573 is a highly selective CDK9 inhibitor that suppresses MCL-1 and induces apoptosis in hematologic cancer cells. Clin. Cancer Res. https://doi.org/10.1158/1078-0432.CCR-19-1853 (2019).

111. Dey, J. et al. Voruciclib, a clinical stage oral CDK9 inhibitor, represses MCL-1 and sensitizes high-risk diffuse large B-cell lymphoma to BCL2 inhibition. Sci. Rep. 7, 18007 (2017).

112. Inoue-Yamauchi, A. et al. Targeting the differential addiction to anti-apoptotic BCL-2 family for cancer therapy. Nat. Commun. 8, 16078 (2017).

113. Alsayegh, K., Matsuura, K., Sekine, H. \& Shimizu, T. Dinaciclib potently suppresses MCL-1 and selectively induces the cell death in human iPS cells without affecting the viability of cardiac tissue. Sci. Rep. 7, 45577 (2017)

114. Prutsch, N. et al. Dependency on the TYK2/STAT1/MCL1 axis in anaplastic large cell lymphoma. Leukemia 33, 696-709 (2019).

115. Maji, S. et al. STAT3- and GSK3beta-mediated Mcl-1 regulation modulates TPF resistance in oral squamous cell carcinoma. Carcinogenesis 40, 173-183 (2019).

116. Yoshimoto, G. et al. FLT3-ITD up-regulates MCL-1 to promote survival of stem cells in acute myeloid leukemia via FLT3-ITD-specific STAT5 activation. Blood 114, 5034-5043 (2009).

117. Shastri, A. et al. Antisense STAT3 inhibitor decreases viability of myelodysplastic and leukemic stem cells. J. Clin. Invest. 128, 5479-5488 (2018).

118. Wang, X. et al. The curcumin analogue hydrazinocurcumin exhibits potent suppressive activity on carcinogenicity of breast cancer cells via STAT3 inhibition. Int. J. Oncol. 40, 1189-1195 (2012).

119. Kiprianova, I. et al. Sorafenib sensitizes glioma cells to the $\mathrm{BH} 3$ mimetic ABT737 by targeting MCL1 in a STAT3-dependent manner. Neoplasia 17, 564-573 (2015).

120. Labisso, W. L. et al. MYC directs transcription of MCL1 and elF4E genes to control sensitivity of gastric cancer cells toward HDAC inhibitors. Cell Cycle 11, 1593-1602 (2012).

121. Trivigno, D., Essmann, F., Huber, S. M. \& Rudner, J. Deubiquitinase USP9x confers radioresistance through stabilization of Mcl-1. Neoplasia 14, 893-904 (2012).

122. Sun, $H$. et al. Bcr-Abl ubiquitination and Usp9x inhibition block kinase signaling and promote CML cell apoptosis. Blood 117, 3151-3162 (2011).

123. Cui, J. et al. EHMT2 inhibitor BIX-01294 induces apoptosis through PMAIP1USP9X-MCL1 axis in human bladder cancer cells. Cancer Cell Int. 15, 4 (2015).

124. Bartholomeusz, G. A. et al. Activation of a novel Bcr/Abl destruction pathway by WP1130 induces apoptosis of chronic myelogenous leukemia cells. Blood 109, 3470-3478 (2007).

125. Kapuria, V. et al. Deubiquitinase inhibition by small-molecule WP1130 triggers aggresome formation and tumor cell apoptosis. Cancer Res. 70, 9265-9276 (2010)
126. Chen, G. et al. Targeting Mcl-1 enhances DNA replication stress sensitivity to cancer therapy. J. Clin. Invest. 128, 500-516 (2018).

127. Turriziani, M. et al. O6-(4-bromothenyl)guanine (PaTrin-2), a novel inhibitor of O6-alkylguanine DNA alkyl-transferase, increases the inhibitory activity of temozolomide against human acute leukaemia cells in vitro. Pharm. Res. 53, 317-323 (2006).

128. Bonfanti, M., Broggini, M., Prontera, C. \& D'Incalci, M. O6-methylguanine inhibits the binding of transcription factors to DNA. Nucleic Acids Res. 19, 5739-5742 (1991).

129. Kwon, Y. T. \& Ciechanover, A. The ubiquitin code in the ubiquitin-proteasome system and autophagy. Trends Biochem. Sci. 42, 873-886 (2017)

130. Hopkins, A. L. \& Groom, C. R. The druggable genome. Nat. Rev. Drug Discov. 1, 727-730 (2002).

131. Sakamoto, K. M. Protacs for treatment of cancer. Pediatr. Res. 67, 505-508 (2010).

132. Gadd, M. S. et al. Structural basis of PROTAC cooperative recognition for selective protein degradation. Nat. Chem. Biol. 13, 514-521 (2017).

133. Guo, J., Liu, J. \& Wei, W. Degrading proteins in animals: "PROTAC"tion goes in vivo. Cell Res. 29, 179-180 (2019).

134. Papatzimas, J. W. et al. From inhibition to degradation: targeting the antiapoptotic protein myeloid cell leukemia 1 (MCL1). J. Med. Chem. 62 5522-5540 (2019).

135. Khan, S. et al. A selective BCL-XL PROTAC degrader achieves safe and potent antitumor activity. Nat. Med. 25, 1938-1947 (2019).

136. He, Y. et al. Using proteolysis-targeting chimera technology to reduce navitoclax platelet toxicity and improve its senolytic activity. Nat. Commun. 11, 1996 (2020).

137. Vick, B. et al. Knockout of myeloid cell leukemia-1 induces liver damage and increases apoptosis susceptibility of murine hepatocytes. Hepatology $\mathbf{4 9}$, 627-636 (2009).

138. Opferman, J. T. et al. Obligate role of anti-apoptotic MCL-1 in the survival of hematopoietic stem cells. Science 307, 1101-1104 (2005).

139. Rinkenberger, J. L., Horning, S., Klocke, B., Roth, K. \& Korsmeyer, S. J. Mcl-1 deficiency results in peri-implantation embryonic lethality. Genes Dev. 14 23-27 (2000).

140. Perciavalle, R. M. \& Opferman, J. T. Delving deeper: MCL-1's contributions to normal and cancer biology. Trends Cell Biol. 23, 22-29 (2013).

141. Thomas, R. L. \& Gustafsson, A. B. MCL1 is critical for mitochondrial function and autophagy in the heart. Autophagy 9, 1902-1903 (2013).

142. Rasmussen, M. L. et al. MCL-1 inhibition by selective $\mathrm{BH} 3$ mimetics disrupts mitochondrial dynamics causing loss of viability and functionality of human cardiomyocytes. iscience 23, 101015 (2020).

143. Opferman, J. T. et al. Development and maintenance of B and T lymphocytes requires antiapoptotic MCL-1. Nature 426, 671-676 (2003).

144. Dzhagalov, I., Dunkle, A. \& He, Y. W. The anti-apoptotic BCl-2 family member Mcl-1 promotes T lymphocyte survival at multiple stages. J. Immunol. 181, 521-528 (2008).

145. Dzhagalov, I., St John, A. \& He, Y. W. The antiapoptotic protein Mcl-1 is essential for the survival of neutrophils but not macrophages. Blood 109, 1620-1626 (2007).

146. Hikita, $\mathrm{H}$. et al. MCl-1 and $\mathrm{BCl}-\mathrm{xL}$ cooperatively maintain integrity of hepatocytes in developing and adult murine liver. Hepatology 50, 1217-1226 (2009).

147. Weber, A. et al. Hepatocyte-specific deletion of the antiapoptotic protein myeloid cell leukemia-1 triggers proliferation and hepatocarcinogenesis in mice. Hepatology 51, 1226-1236 (2010).

148. Kelly, G. L. et al. Targeting of MCL-1 kills MYC-driven mouse and human lymphomas even when they bear mutations in p53. Genes Dev. 28, 58-70 (2014).

149. Grabow, S. et al. Critical B-lymphoid cell intrinsic role of endogenous MCL-1 in C-MYC-induced lymphomagenesis. Cell Death Dis. 7, e2132 (2016).

150. Harrigan, J. A., Jacq, X., Martin, N. M. \& Jackson, S. P. Deubiquitylating enzymes and drug discovery: emerging opportunities. Nat. Rev. Drug Discov. 17, 57-78 (2018). 